\title{
Improved Surgical Outcomes for Breast Cancer Patients Receiving Neoadjuvant Aromatase Inhibitor Therapy: Results from a Multicenter Phase II Trial
}

\author{
John A Olson Jr, MD, PhD, FACS, G Thomas Budd, MD, Lisa A Carey, MD, Lyndsay A \\ Harris, MD, Laura J Esserman, MD, MBA, FACS, Gini F Fleming, MD, Paul K Marcom, MD, \\ George S Leight Jr, MD, FACS, Therese Giuntoli, BA, CCRP, Paul Commean, BA, Kyongtae \\ Bae, MD, Jingqin Luo, PhD, and Matthew J Ellis, MB, BChir, PhD \\ Departments of Surgery; (Olson, Leight) and Medicine (Marcom), Duke Comprehensive Cancer \\ Center, Durham, NC; Dana Farber Cancer Center, Boston, MA(Harris); Cleveland Clinic Taussig \\ Cancer Center, Cleveland, OH (Budd); Lineberger Cancer Center, University of North Carolina, \\ Durham, NC (Carey); University of California San Francisco and Helen Diller Family \\ Comprehensive Cancer Center, San Francisco, CA (Esserman); University of Chicago Cancer \\ Research Center, Chicago, IL (Fleming); Department of Medicine, Division of Oncology; (Giuntoli, \\ Ellis),Mallinckrodt Institute of Radiology (Commean, Bae), and Department of Biostatistics (Luo), \\ Washington University School of Medicine, St Louis, MO; and Siteman Comprehensive Cancer \\ Center, St Louis, MO (Ellis)
}

\begin{abstract}
BACKGROUND-Neoadjuvant aromatase inhibitor therapy has been reported to improve surgical outcomes for postmenopausal women with clinical stage II or III hormone receptorpositive breast cancer. A multicenter phase II clinical trial was conducted to investigate the value of this approach for US surgical practice.
\end{abstract}

STUDY DESIGN-One hundred fifteen postmenopausal women with $>2 \mathrm{~cm}$, estrogen receptor (ER) or progesterone receptor (PgR)-positive breast cancer were enrolled in a trial of 16 to 24 weeks of letrozole $2.5 \mathrm{mg}$ daily before operation.

\begin{abstract}
RESULTS-One hundred six patients were eligible for primary analysis, 96 underwent operations, 7 received chemotherapy after progressive disease, and 3 did not undergo an operation. Baseline surgical status was marginal for breast-conserving surgery (BCS) in 48 (45\%), 47 were definitely ineligible for BCS (44\%), and 11 were inoperable by standard mastectomy (10\%). Overall Response Evaluation Criteria In Solid Tumors clinical response rate in the breast was $62 \%$, with $12 \%$ experiencing progressive disease. Fifty percent underwent BCS, including 30 of $46(65 \%)$ patients who were initially marginal for BCS and 15 of $39(38 \%)$ patients who were initially ineligible for BCS. All 11 inoperable patients successfully underwent operations,
\end{abstract}

\footnotetext{
(C) 2009 by the American College of Surgeons

Correspondence address: Matthew J Ellis, MD, Washington University, Siteman Cancer Center, Campus Box 8056, $660 \mathrm{~S}$ Euclid Ave, St Louis, MO 63110.mellis@dom.wustl.edu.

Disclosure Information: All other authors have nothing to disclose.

Presented at the Southern Surgical Association 120th Annual Meeting, West Palm Beach, FL, December 2008.

Author Contributions

Study conception and design: Olson, Ellis

Acquisition of data: Olson, Budd, Carey, Harris, Esserman, Fleming, Marcom, Leight, Ellis

Analysis and interpretation of data: Olson, Giuntoli, Commean, Bae, Luo, Ellis

Drafting of manuscript: Olson, Ellis

Critical revision: Olson, Giuntoli, Luo, Ellis
} 
including 3 (27\%) who had BCS. Nineteen percent of patients undergoing mastectomy had a pathologic T1 tumor, suggesting that some highly responsive tumors were overtreated surgically.

CONCLUSIONS-Neoadjuvant aromatase inhibitor improves operability and facilitates BCS, but there was considerable variability in responsiveness. Better techniques to predict response, determine residual tumor burden before operation, and greater willingness to attempt BCS in responsive patients could additionally improve the rate of successful BCS.

Randomized trials of neoadjuvant chemotherapy against immediate operation have been shown to increase the rate of breast-conserving surgery (BCS) without compromising survival. ${ }^{1-3}$ For the approximately $75 \%$ of patients with tumors that express estrogen receptor (ER), neoadjuvant endocrine therapy is a logical alternative., ${ }^{4}$ This is particularly the case for postmenopausal women with $\mathrm{ER}^{+}$disease, where tamoxifen provides at least twice the adjuvant treatment benefit of chemotherapy, and the response to neoadjuvant chemotherapy, in terms of the pathologic complete response rate, is low. 6,7

The practice of treating patients with inoperable breast cancer with stilbestrol was established $>50$ years ago. ${ }^{8,9}$ After stilbestrol was replaced by tamoxifen in the early 1980s, use of tamoxifen before operation continued to be explored for older patients with locally advanced disease. ${ }^{10}$ Most recently, third-generation aromatase inhibitors have replaced tamoxifen for this indication because there is evidence for greater efficacy as both neoadjuvant and as adjuvant treatment. ${ }^{11-15}$ Neoadjuvant aromatase inhibitor studies have documented objective response rates of between $37 \%$ and $60 \%$ and conversion from mastectomy to BCS in up to $50 \%$ of patients. ${ }^{12-15}$ In these studies, progression of disease typically occurred in $₫ 0 \%$ of patients. Recently, a prognostic algorithm, the Preoperative Endocrine Prognostic Index, has been developed, which incorporates information on Ki67, ER, and stage derived from the postneoadjuvant endocrine therapy tumor specimen to identify groups of patients with such a low relapse rate that they can consider foregoing adjuvant chemotherapy. ${ }^{16}$ These data establish that the benefits of neoadjuvant endocrine therapy are very similar to neoadjuvant chemotherapy, ie, not only improving surgical outcomes, but determining "on treatment" prognosis based on the response of the primary tumor to neoadjuvant therapy. The formal clinical experience of neoadjuvant endocrine therapy in the US has been largely limited to smaller single-institution series or the contribution of a modest number of patients to international phase III trials. ${ }^{13,17} \mathrm{We}$ performed a multicenter, phase II trial within the US using the nonsteroidal inhibitor letrozole in patients who were either marginal candidates or not candidates for BCS. In this article, we discuss surgical outcomes in this study, with an emphasis on factors associated with successful breast-conservation therapy.

\section{METHODS}

\section{Study design}

The study was an open-label, multicenter phase II trial in which eligible patients were assigned to receive oral letrozole (Femara; Novartis Pharmaceuticals), $2.5 \mathrm{mg}$ daily for 16 to 24 weeks before surgical therapy from commercial stock. Ethics committee approval was obtained at all participating sites and the trial is registered with the National Cancer Institute clinical trials database as NCT00084396 (http://www.cancer.gov/clinicaltrials). The clinical objectives of the study were to document the response rate to 4 months of neoadjuvant letrozole therapy by clinical and radiologic measurements; to document rate of improvement in surgical outcomes; to document longterm outcomes of preoperative neoadjuvant letrozole; and to document the safety of preoperative neoadjuvant letrozole. 


\section{Patients}

Eligible patients were postmenopausal women with previously untreated clinical stage II and III ER- or progesterone receptor (PgR)-positive breast cancer with a good performance status (Eastern Cooperative Oncology Group performance status 0 to 2).The tumor must have been palpable and $>2 \mathrm{~cm}$ in size on clinical measurement. Postmenopausal status was defined as amenorrhea for at least 1 year, bilateral surgical oophorectomy, or folliclestimulating hormone and estradiol in the postmenopausal range. Concurrent hormone replacement therapy or other endocrine agents were not allowed.

\section{Clinical assessment}

Clinical response was determined monthly with bidimensional caliper measurement of the primary tumor and clinical staging of the axilla by physical examination. Baseline surgical status was determined by the treating surgeon before treatment and classified as inoperable, marginal candidate for BCS, or ineligible for BCS. Classification of baseline surgical status was based on surgeon judgment; no a priori criteria were formulated. Response Evaluation Criteria in Solid Tumors (RECIST) criteria were used for primary reporting of data, but WHO response data were also recorded when a second dimension was available from the case report form.

\section{Imaging}

All consented patients were required to undergo pretreatment imaging with mammography and ultrasonography with repeat imaging at the 16-week visit. Followup imaging was required if clinical progression was documented on clinical examination before the presurgical visit at week 16. Mammograms were reviewed at the local institution and, when available, centrally. Patients were also excluded from the central radiographic analysis when different views were provided that were not comparable. The cranial-caudal and mediallateral oblique mammogram films, both pretreatment and 16 weeks posttreatment, were digitized and saved as Digital Imaging and Communications in Medicine (DICOM) files. In a small number of patients, a medial-lateral view was used when a medial-lateral oblique view was not available. The Digital Imaging and Communications in Medicine files were uploaded into the Analyze software system (Mayo Clinic, Biomedical Imaging Resource) for performing RECIST measurements. ${ }^{18}$ An experienced radiologist (KB) measured the longest dimension of the lesion in the cranial-caudal and medial-lateral oblique views for pre- and posttreatment by using Image Edit (Analyze software tool) to define the perimeter of the lesion semiautomatically. In some patients, the program delineated the boundary well, but in other patients manual editing of the region was required to remove areas not identified as lesion or to add areas of lesion.

\section{Biomarker assessment}

Diagnosis of invasive breast cancer was made by core needle biopsy. Tumor ER status was determined at accruing sites according to local assays. Assignment of ER-positive or progesterone receptor positive was defined as $\geq 10 \%$ nuclear staining of the invasive component of the tumor. Similarly, HER2 data were collected from local testing results.

\section{Operation}

Operations were performed after 16 to 18 weeks of letrozole therapy, except for 14 patients with partial response (PR) at 16 weeks, for whom an extension of up to 24 weeks was permitted. Letrozole was continued until the day before operation. Choice and conduct of operation to the breast and axilla was not prescribed by the protocol and was left to the judgment of the operating surgeon and preference of the patient. Final surgical outcomes were reported for BCS versus mastectomy. 


\section{Statistical analysis}

Analysis of categorical factors associated with surgical out-comes was performed using chisquare tests or Fisher's exact test with fewer than five cell counts (for marginally BCS eligible, not BCS eligible, and not operable by standard mastecttomy). For $2 \times 2$ contingency tables, relative risks and $95 \%$ confidence intervals were given for mastectomy outcomes. Wilcoxon signed rank test was used to compare repeated measurements (pre- and postmeasurements) and also two related measurements. Waterfall plots were generated for visualization of the relationship between a sorted continuous feature of mammographic and clinical response and surgical outcomes. All statistical tests were two-sided and a p value $₫) .05$ was considered significant. Factors with a significant $p$ value $₫ 0.2$ from contingency table analysis with mastectomy outcomes were selected for multivariate logistic regression. Chi-square $\mathrm{p}$ values on regression coefficients and odds ratios accompanied with $95 \%$ Wald confidence intervals were reported.

\section{RESULTS}

\section{Study population}

Information on the patient population and progress of patients through the protocol is provided in Table 1 and Figure 1. Median patient age was 67 years (range 48 to 89 years) and the majority (94\%) had good performance status (Eastern Cooperative Oncology Group performance status 0 or 1), indicating that most patients were not burdened by a high degree of comorbidity. Initial tumor size was large (median $4.9 \mathrm{~cm}$, range 2.3 to $12 \mathrm{~cm}$ ) by caliper measurement and was most often of ductal histology (56\%), with the remainder recorded as lobular (24\%) or mixed (17\%). One hundred fifteen patients were enrolled and 106 constituted the per-protocol population. Ninety-two underwent operations around the 16th week, and 14 were treated on a protocol-defined optional treatment extension to the 24th week for those who were partial responders at 16 weeks. Ten patients did not have immediate operation after letrozole treatment; seven received neoadjuvant chemotherapy, two had comorbidities that precluded operation, and one refused operation.

\section{Toxicity and adverse events}

Toxicities and intolerances were typical for aromatase inhibitor therapy and there were no serious unexpected adverse events that were considered likely to be study-drug-related (Table 2). No patient withdrew from the study because of toxicity. There were no studyrelated deaths.

\section{Clinical response}

Clinical response of the primary tumor to letrozole was assessed by caliper examination and classified by RECIST criteria in 100 patients (6 patients had inadequate data) (Table 3 ). Thirteen (13\%) of the patients experienced a complete response (CR), 49 (49\%) had PR, and $26(26 \%)$ had stable disease. Twelve (12\%) patients experienced clinical progressive disease. Patients with progressive disease received either neoadjuvant chemotherapy $(n=7)$ or had immediate operation $(n=5)$. Adequate bidimensional measurements for calculation of response by WHO criteria were obtained in 83 patients and produced very similar results (Table 2). Median clinical tumor size after letrozole treatment was significantly smaller than pretreatment tumor size ( 4.9 versus $3.0 \mathrm{~cm}$; $\mathrm{p}=0.0001$ ), as expected.

\section{Radiologic response}

Radiologic response determined by ultrasonography and mammography was examined in subsets of patients according to data availability (Table 4). Response to letrozole recorded by local mammography assessment was available in 56 patients, producing an overall 
response rate $(\mathrm{CR}+\mathrm{PR})$ of $38 \%$. Overall ultrasonography response rate in 77 patients was similar (38\%). The incomplete radiographic response documentation was investigated more with a post hoc central analysis that was conducted on 63 pre- and posttherapy mammograms. Films were digitized (unless already digital) and an image analyzer was used to define the borders of the tumor and a longest dimension was determined. The central review documented an overall RECIST response of 34\%, which was similar to local mammography response data; the correlation between the percentage decreases recorded by local versus central mammography RECIST measurements was low (Spearman correlation coefficient $0.34 ; \mathrm{p}=0.036$ ). This might have been, in part, because it was not possible to determine which views the local radiologists used for response assessment. It might be noteworthy that local mammographic assessment of CR (9\%) and progressive disease (16\%) was more closely aligned with clinical measurement data, but these assessments might be biased if the radiologist was aware of the clinician's assessment of response.

\section{Surgical outcomes}

Forty-eight of the 96 patients (50\%) who underwent operations had successful BCS. This group included $30(65 \%)$ who were deemed as marginal for BCS at presentation, $15(38 \%)$ of 39 patients deemed ineligible for BCS, and $3(27 \%)$ of 11 patients deemed inoperable by their surgeon based on pretreatment assessments. Of the seven patients who had disease progression necessitating neoadjuvant chemotherapy before operation, three ultimately had BCS and four had mastectomy. There was marked variability in the BCS rate between the two highest accruing centers, with a $29 \%$ BCS rate recorded in one and $65 \%$ in the other. This difference cannot be explained by pre-treatment or posttreatment clinical size because there were no significant differences, but in the site with the higher mastectomy rate the pathologic T size was, on average, higher $(3.7 \mathrm{~cm}$, range $1-12.2 \mathrm{~cm}$ versus $2.1 \mathrm{~cm}$, range $1.9,0-5.5 \mathrm{~cm} ; \mathrm{p}=0.003$ ). Differences in surgical practice about selection of patients for breast conservation was not necessarily the major factor in determining the twofold difference in BCS rates between the two centers.

\section{Pathologic results}

Pathologic staging results (Table 5) supported the conclusion that a number of patients experienced substantial tumor downstaging with letrozole treatment. Median pathologic tumor size was $2.1 \mathrm{~cm}$ (range 0.0 to $12.2 \mathrm{~cm}$ ), which was low considering the patients entered the study with clinical stage II or III breast cancer. Clinical response rate (CR + PR) was $84 \%$ ( 32 of 38) in the pathologic stage I or 0 group versus $55 \%$ (29 of 53) in the pathologic stage II or III group (response rate difference $=29 \%$; $95 \%$ CI, 11.8 to $47.2 \%$; $p=$ 0.003 ). Downstaging of the axilla could not be adequately assessed in this study because baseline node status was not systematically evaluated. The histologic subtypes of breast cancer (ductal versus lobular versus mixed) were largely unchanged with treatment, but there was a significant improvement in the proportion of favorable histologic grade (postversus pretreatment tumor samples, respectively); grade 1 in 30 (32\%) versus $21(23 \%)$, grade 2 in $57(60 \%)$ versus $58(55 \%)$, and grade 3 in $8(8 \%)$ versus $22(23 \%)$ pre- and posttreatment samples $(\mathrm{p}=0.0014)$.

\section{Factors associated with breast conservation}

To identify factors that might be used to guide the selection of patients for breast conservation, pathologic T stage was examined by operation type (Fig. 2). Not surprisingly,

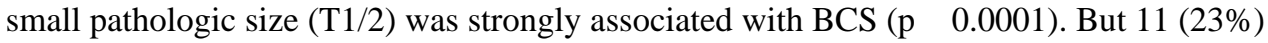
of 46 patients who had mastectomy had pathologic T stage I tumors (Fig. 2), indicating they might have been overtreated surgically (patient choice about operation was not recorded in the study). In addition, a similar proportion of patients with T2 tumors had BCS (18 of 46; $38 \%$ ) as had mastectomy ( 21 of $46 ; 44 \%$ ), suggesting that many more women could have 
had BCS. To present the complex relationships between clinical RECIST responses, pathologic stage, and surgical outcomes, waterfall plots were constructed (Fig. 3). These data illustrate how patients who received BCS experienced greater decreases in tumor size by clinical examination (Fig. 3A), but reveal individual patients with highly responsive tumors and low pathologic stage who nonetheless had a mastectomy. An examination of waterfall plots comparing tumor response by ultrasonography (Fig. 3B) and central mammography (Fig. 3C) with final pathologic T stage and surgical outcomes showed less evidence of clear relationships between imaging response and pathologic size. These data suggest that clinical examination remains the most important modality for assessing response to treatment. To more formally identify factors associated with the surgical approach undertaken, univariate and multivariable analyses were performed (Table 6,7). Univariate analysis revealed the following factors were significantly associated with mastectomy: pre-aromatase inhibitor clinical T3/4 stage versus lower stage (relative risk, $2.4 ; 95 \%$ CI, 1.5 to $3.69 ; \mathrm{p}<0.0001$ ), BCS ineligible/inoperable surgical status versus marginal candidate for BCS (relative risk, $1.84 ; 95 \%$ CI, 1.18 to 2.88 ; $\mathrm{p}=0.0042$ ), postaromatase inhibitor clinical T2/3/4 stage versus lower clinical stage (T1/0) (relative risk, 2.3; 95\% CI, 1.26 to $4.10 ; \mathrm{p}=0.0013$ ). Clinical response (relative risk, $1.49 ; 95 \% \mathrm{CI}, 1.0$ to $2.2 ; \mathrm{p}$ $=0.06$ ) was not significant, suggesting it was not response per se, but final clinical stage that was the most critical factor in the decision to attempt BCS. Pathologic T3/4 stage (relative risk, 2.3; 95\% CI, 1.73 to $3.11 ; \mathrm{p}<0.0001$ ) and $\mathrm{N} 1$ to 3 stage (relative risk, 2.3014; 95\% CI, 1.3467 to $3.9330 ; p=0.0004$ ) were also highly significantly associated with mastectomy, confirming that this procedure was mostly a consequence of extensive residual disease. Factors with $\mathrm{p}$ values $<0.2$ were entered into a multivariate logistic regression model to identify factors that were independently associated with mastectomy. Not surprisingly, baseline tumor size $(\mathrm{T} 3 / 4$ versus $\mathrm{T} 2, \mathrm{p}=0.012)$ and surgical status at baseline (other versus marginal candidate for BCS, $p=0.019$ ) were the most significant factors, but week-16 clinical stage $\mathrm{T} 1 / 0$ was also a factor that remained significant in the multivariate model $(\mathrm{T} 2 / 3 / 4$ vs T1/0, $\mathrm{p}=0.029)$. Because there were no patients in this clinical stage category at baseline, this observation supports the notion that tumor response promotes breast conservation.

Information on breast cancer recurrences with a median followup of 36 months (range 5 to 84 months) is available for the 106 patients. To date, eight patients have experienced distant recurrence either alone $(n=6)$ or with concurrent local recurrence in two mastectomy patients. Six of the eight died of recurrent breast cancer. Five patients have died of other causes. Eighty-three of 96 patients are currently alive without recurrence.

\section{DISCUSSION}

Clinical response rate in this study (62\%) compares favorably with the performance of the aromatase inhibitor arms of several international phase III trials of neoadjuvant endocrine therapy, including the letrozole arm of the P024 trial (55\%), and anastrozole arms of the Immediate Preoperative Anastrozole, Tamoxifen, or Combined with Tamoxifen (IMPACT) (37\%) and Preoperative Arimidex Compared with Tamoxifen (PROACT) (49.7\%) trials. ${ }^{12,13,15}$ Risk of clinical disease progression during letrozole therapy in this trial was $12 \%$, which was slightly higher than that reported in the letrozole P024 trial $(8 \%) .{ }^{13}$ This finding emphasizes the need for careful patient selection and better predictive tests, but salvage with neoadjuvant chemotherapy or immediate operation is a readily available approach for this relatively uncommon situation.

At the other end of the clinical response spectrum are those patients whose final clinical stage is T1/0. The data we have presented suggest that these patients should be offered breast conservation even if the imaging results are equivocal, because many of these patients 
have a favorable pathologic stage category at time of operation. Imaging approaches with either ultrasonography or mammography were disappointing decision-making tools in this series, with poor relationships with final pathologic size and inconsistent adherence by investigators with protocol requirements (Table 3, Fig. 3B, C). It is conceivable that these tests might have even been misleading in certain patients, leading to inappropriate mastectomies in patients with minimal residual disease (Fig. 2). Data presented in Figure 3 suggest that radiologic images can overestimate the amount of residual disease, perhaps because there is no clear discrimination between persistently dense but tumor-free fibrotic tissue typical of successful treatment and tissue that contains persistent cancer. In addition, it might be noteworthy that several extreme responders by digital mammography had stage I disease but underwent mastectomy (Fig. 3C). In individual patients, a careful review of the mammogram, perhaps using image analysis software, might be helpful. Ultrasonography has previously been reported to be an accurate predictor of response to neoadjuvant aromatase inhibitor therapy, but this article reflects the experience of a single center and predominantly that of a single surgeon. ${ }^{14}$ In multicenter studies, there is much less consistency in ultrasonography technique and a single patient can be examined by several radiologists. Reports on difficulties with imaging response to neoadjuvant chemotherapy are similar to the experience we report here, suggesting that poor correlation between pathologic response and imaging response is a generic problem. ${ }^{19}$ MRI was not formally studied in this investigation. The increased sensitivity of MRI must be balanced against the propensity of MRI to overestimate the size of breast cancer. MRI will not necessarily improve surgical decision making after neoadjuvant endocrine therapy. ${ }^{20}$ In the final analysis, clinical documentation of tumor regression and an attempt at breast conservation with intraoperative assessment of margins when necessary is likely to remain the standard of care until imaging approaches improve.

Clinical outcomes from this trial unequivocally demonstrate that the benefits of neoadjuvant aromatase inhibitor therapy extend to patients receiving treatment in US surgical practice. Multivariate analysis (Table 7) indicates that there is no reason to discriminate against younger postmenopausal women or patients with lobular carcinoma because these factors did not impact on the incidence of successful conversion to breast conservation. At a median followup of 3 years, it is reassuring to note the absence of local recurrence in patients experiencing BCS. Given the indolent biology of ER-positive disease, longer followup will be required to be certain about the local recurrence rate in this clinical setting.

Currently, a randomized trial comparing the three FDA approved aromatase inhibitors is being conducted by the American College of Surgeons Oncology Group (ACOSOG Z1031) to identify the most effective aromatase inhibitor in this setting. Results we report here support continued use of clinical response by RECIST criteria as the primary endpoint, although the number of patients in the clinical stage I or 0 (complete response) category, the rate of breast conservation, and the final pathologic stage are all appropriate additional informative endpoints.

In conclusion, our phase II experience with letrozole confirms the value of neoadjuvant endocrine therapy for postmenopausal women with ER-rich tumors that require downstaging to improve surgical outcomes. Additional investigation of this approach is warranted and consistent benefits in terms of an improvement in surgical outcomes across multiple studies indicate that neoadjuvant aromatase inhibitor should be considered a standard of care in the appropriate patient population. 


\title{
Acknowledgments
}

Dr Ellis received honoraria, consulting fees and research grants from both Novartis and AstraZeneca as speaker, ad hoc consultant, and scientist on grants, and a consulting fee from Pfizer as an ad hoc consultant. Dr Budd received a research grant as investigator and a consulting fee is an advisory board member for Novartis, and received a fee as an ad hoc consultant for Pfizer. Dr Marcom is a consulting independent contractor for AstraZeneca Pharmaceuticals LP; and received grant support and consulting fees as investigator and contract teaching for Novartis.

Supported by National Cancer Institute (NCI) grant R01 CA095614 and Avon NCI Partners in Progress Award 3P50 CA68438-07S2.

\section{Abbreviations and Acronyms}

\author{
BCS breast-conserving surgery \\ CR complete response \\ ER estrogen receptor \\ PR partial response \\ RECIST Response Evaluation Criteria in Solid Tumors
}

\section{REFERENCES}

1. Wolmark N, Wang J, Mamounas E, et al. Preoperative chemotherapy in patients with operable breast cancer: nine-year results from National Surgical Adjuvant Breast and Bowel Project B-18. J Natl Cancer Inst Monogr. 2001:96-102. [PubMed: 11773300]

2. Bear HD, Anderson S, Smith RE, et al. Sequential preoperative or postoperative docetaxel added to preoperative doxorubicin plus cyclophosphamide for operable breast cancer: National Surgical Adjuvant Breast and Bowel Project Protocol B-27. J Clin Oncol. 2006; 24:2019-2027. [PubMed: 16606972]

3. Rastogi P, Anderson SJ, Bear HD, et al. Preoperative chemotherapy: updates of National Surgical Adjuvant Breast and Bowel Project Protocols B-18 and B-27. J Clin Oncol. 2008; 26:778-785. [PubMed: 18258986]

4. Barnes DM, Millis RR, Gillett CE, et al. The interaction of oestrogen receptor status and pathological features with adjuvant treatment in relation to survival in patients with operable breast cancer: a retrospective study of 2660 patients. Endocr Relat Cancer. 2004; 11:85-96. [PubMed: 15027887]

5. Ma CX, Ellis MJ. Neoadjuvant endocrine therapy for locally advanced breast cancer. Semin Oncol. 2006; 33:650-656. [PubMed: 17145344]

6. Effects of chemotherapy and hormonal therapy for early breast cancer on recurrence and 15-year survival: an overview of the randomised trials. Lancet. 2005; 365:1687-1717. [PubMed: 15894097]

7. Colleoni M, Viale G, Zahrieh D, et al. Chemotherapy is more effective in patients with breast cancer not expressing steroid hormone receptors: a study of preoperative treatment. Clin Cancer Res. 2004; 10:6622-6628. [PubMed: 15475452]

8. Haddow A, Watkinson JM, Paterson E. Influence of synthetic oestrogens upon advanced malignant disease. BMJ. 1944; 2:393-398. [PubMed: 20785660]

9. Kennedy BJ, Kelley RM, White G, Nathanson IT. Surgery as an adjunct to hormone therapy of breast cancer. Cancer. 1957; 10:1055-1075. [PubMed: 13472656]

10. Ellis MJ. Preoperative endocrine therapy for older women with breast cancer: renewed interest in an old idea. Cancer Control. 2000; 7:557-562. [PubMed: 11088064]

11. Howell A, Cuzick J, Baum M, et al. Results of the ATAC (Arimidex, Tamoxifen, Alone or in Combination) trial after completion of 5 years' adjuvant treatment for breast cancer. Lancet. 2005; 365:60-62. [PubMed: 15639680]

12. Smith IE, Dowsett M, Ebbs SR, et al. Neoadjuvant treatment of postmenopausal breast cancer with anastrozole, tamoxifen, or both in combination: the Immediate Preoperative Anastrozole, 
Tamoxifen, or Combined with Tamoxifen (IMPACT) multicenter double-blind randomized trial. J Clin Oncol. 2005; 23:5108-5116. [PubMed: 15998903]

13. Eiermann W, Paepke S, Appfelstaedt J, et al. Preoperative treatment of postmenopausal breast cancer patients with letrozole: a randomized double-blind multicenter study. Ann Oncol. 2001; 12:1527-1532. [PubMed: 11822750]

14. Dixon JM, Renshaw L, Bellamy C, et al. The effects of neoadjuvant anastrozole (Arimidex) on tumor volume in postmenopausal women with breast cancer: a randomized, double-blind, singlecenter study. Clin Cancer Res. 2000; 6:2229-2235. [PubMed: 10873072]

15. Cataliotti L, Buzdar AU, Noguchi S, et al. Comparison of anastrozole versus tamoxifen as preoperative therapy in postmenopausal women with hormone receptor-positive breast cancer: the pre-operative "Arimidex" compared to Tamoxifen (PROACT) trial. Cancer. 2006; 106:20952103. [PubMed: 16598749]

16. Ellis MJ, Tao Y, Luo J, et al. Outcome prediction for estrogen receptor-positive breast cancer based on postneoadjuvant endocrine therapy tumor characteristics. J Natl Cancer Inst. 2008; 100:1380-1388. [PubMed: 18812550]

17. Hoff PM, Valero V, Buzdar AU, et al. Combined modality treatment of locally advanced breast carcinoma in elderly patients or patients with severe comorbid conditions using tamoxifen as the primary therapy. Cancer. 2000; 88:2054-2060. [PubMed: 10813717]

18. Robb R. The biomedical imaging resource at Mayo Clinic. IEEE Trans Med Imaging. 2001; 20:854-867. [PubMed: 11585203]

19. Chagpar AB, Middleton LP, Sahin AA, et al. Accuracy of physical examination, ultrasonography, and mammography in predicting residual pathologic tumor size in patients treated with neoadjuvant chemotherapy. Ann Surg. 2006; 243:257-264. [PubMed: 16432360]

20. Berg WA, Gutierrez L, NessAiver MS, et al. Diagnostic accuracy of mammography, clinical examination, US, and MR imaging in preoperative assessment of breast cancer. Radiology. 2004; 233:830-849. [PubMed: 15486214] 


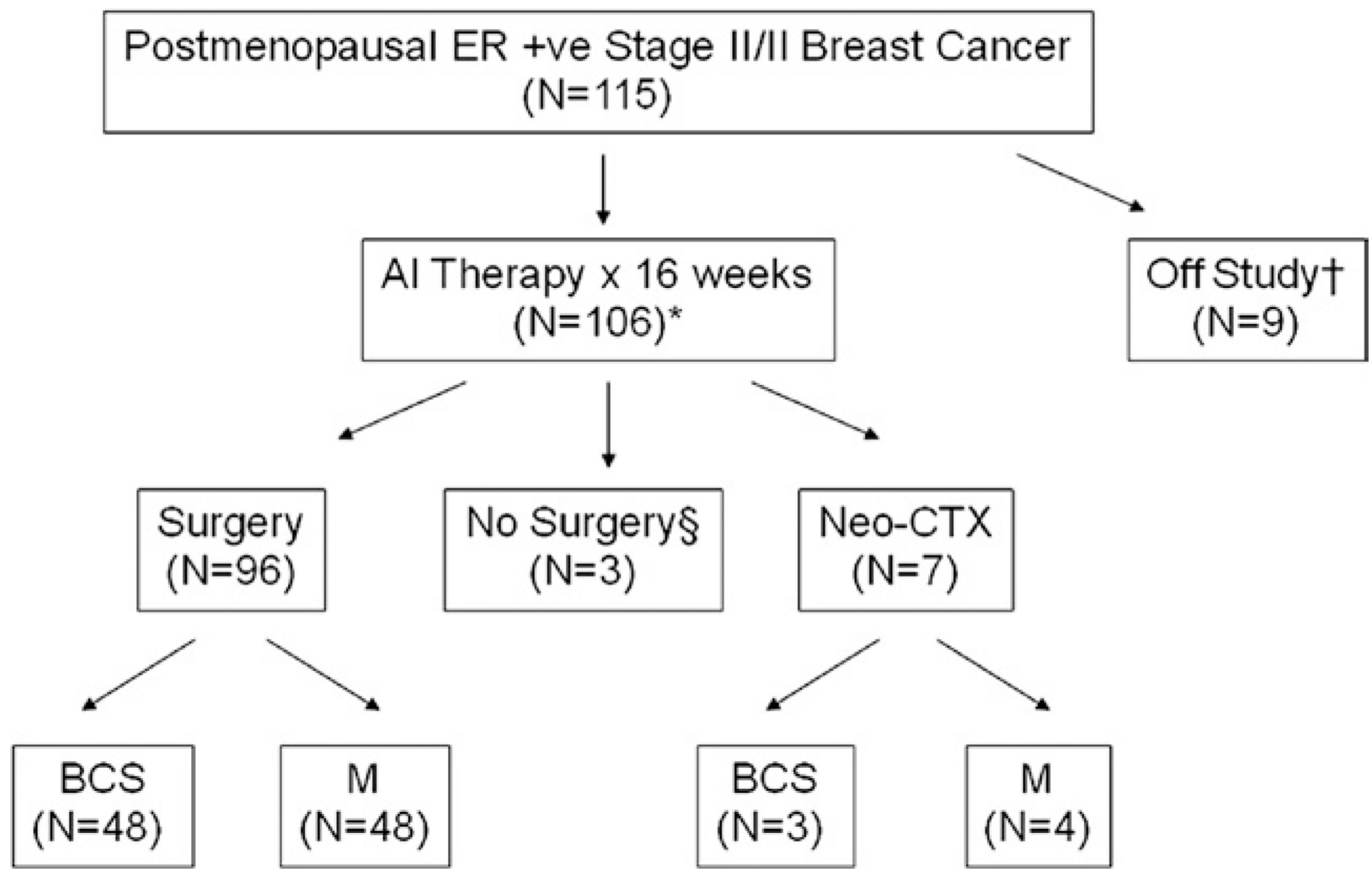

Figure 1.

This Consolidated Standards of Reporting Trials (CONSORT)-type diagram summarizes patient progress though the protocol. $\dagger$ Six patients withdrew consent, two patients were ineligible on additional investigation and one patient died before receipt of study drug. *Fourteen patients were treated on a protocol defined extension to 24 weeks for patients experiencing a partial response at 16 weeks who were thought to potentially benefit from additional tumor regression. ${ }^{\S}$ One patient refused operation and two patients did not have operations because of severe intercurrent illness unrelated to study drug. AI, aromatase inhibitor; BCS, breast-conserving surgery; CTX, chemotherapy treatment; ER, estrogen receptor; $\mathrm{M}$, mastectomy. 


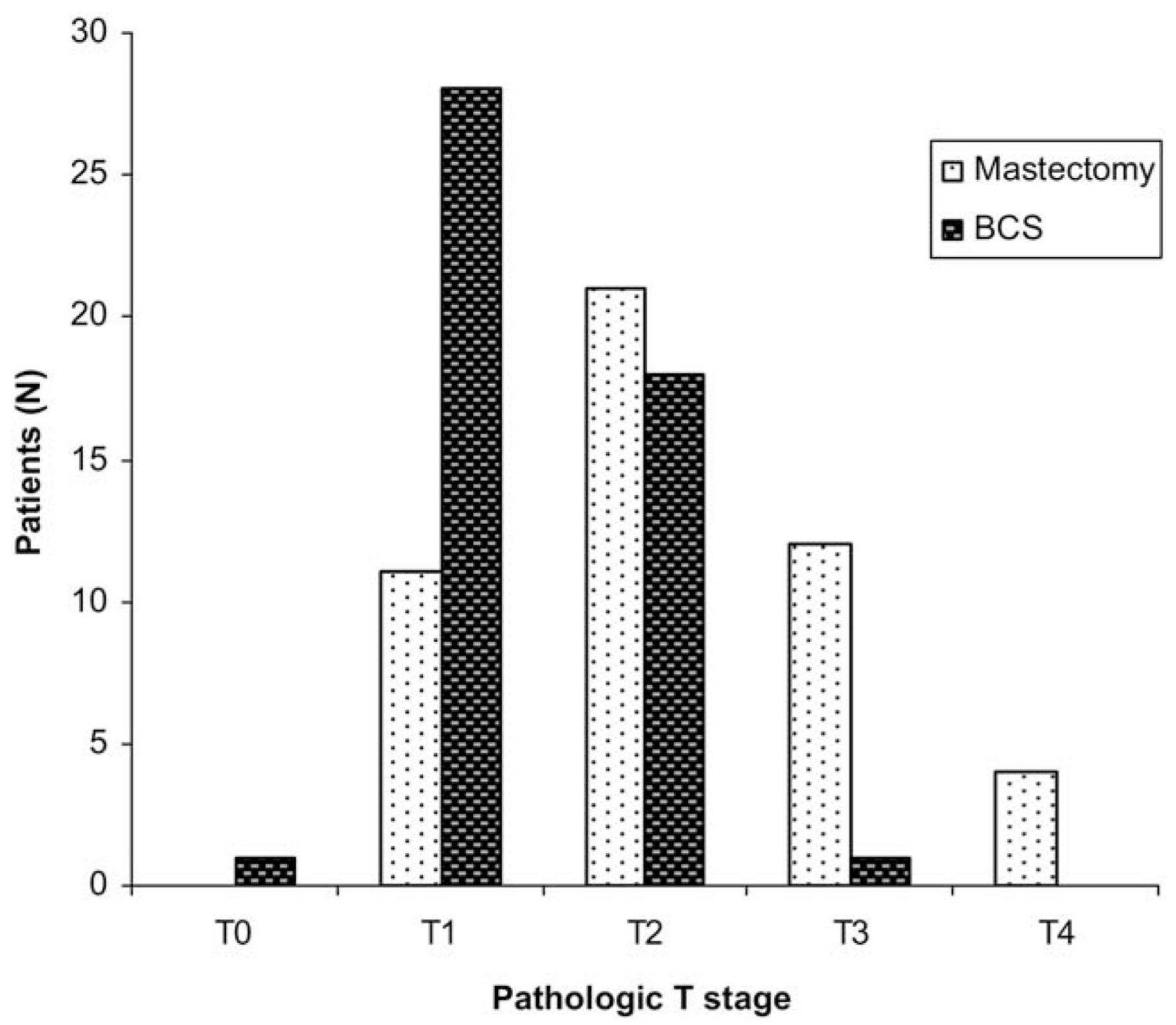

Figure 2.

Pathologic T stage for neoadjuvant aromatase inhibitor treated patients $(n=96)$ separated by operative outcomes. Ninety-six patients had operations after neoadjuvant endocrine therapy alone, of whom 48 had breast-conserving surgery (BCS) (dark bars) and 48 had mastectomy (light bars). Pathologic stage was determined by American Joint Committee on Cancer criteria. 

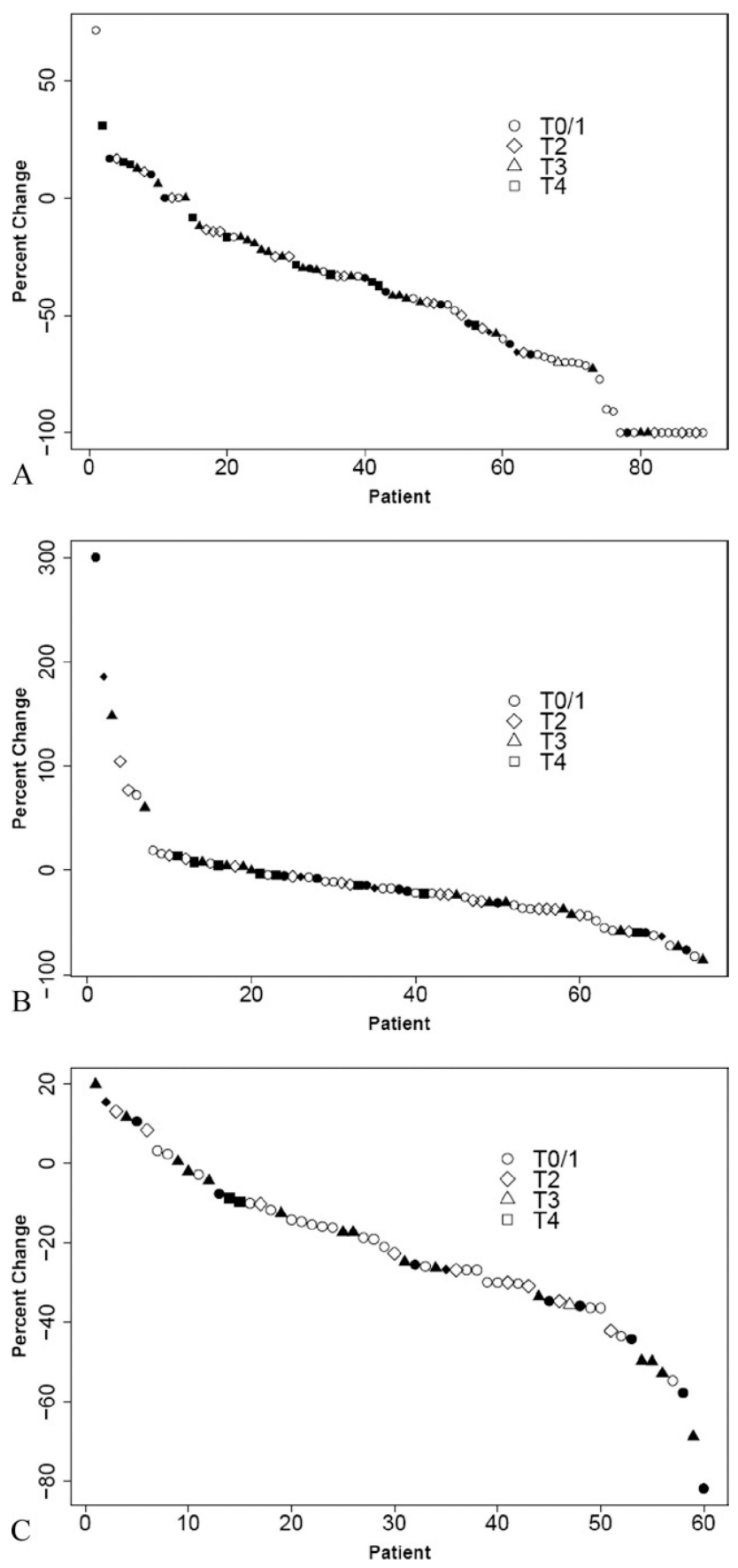

Figure 3.

Waterfall plots for (A) clinical response (Response Evaluation Criteria in Solid Tumors [RECIST]), (B) ultrasonography response RECIST, and (C) mammography RECIST response from central measurements. Response data, as percentage change from baseline are plotted as a sorted continuous feature. Solid symbols, patients who underwent mastectomy; open symbols, patients who underwent breast conservation; circles, pathologic T1/0 disease; diamond T2 disease; triangle T3; square T4. 
Table 1

Patient Characteristics, Tumor Stage, Tumor Grade, Tumor Type, and Biomarker Status $(\mathrm{n}=106)$

\begin{tabular}{|c|c|}
\hline Characteristic & Value \\
\hline Age (y), median (range) & $67(48-89)$ \\
\hline \multicolumn{2}{|l|}{ ECOG performance status, n (\%) } \\
\hline 0 & $78(74)$ \\
\hline 1 & $22(21)$ \\
\hline 2 & $6(6)$ \\
\hline \multicolumn{2}{|l|}{ Ethnicity, n (\%) } \\
\hline Caucasian & $74(70)$ \\
\hline African American & $24(23)$ \\
\hline Hispanic & $6(5)$ \\
\hline Asian & $1(1)$ \\
\hline Unknown & $1(1)$ \\
\hline Clinical tumor size $(\mathrm{cm})$, median (range) & $4.9(2.3-12.0)$ \\
\hline \multicolumn{2}{|l|}{ T stage, $\mathrm{n}(\%)$} \\
\hline $\mathrm{T} 2$ & $56(53)$ \\
\hline $\mathrm{T} 3$ & $39(37)$ \\
\hline $\mathrm{T} 4$ & $11(10)$ \\
\hline \multicolumn{2}{|l|}{$\mathrm{N}$ stage, $\mathrm{n}(\%)$} \\
\hline No & $66(62)$ \\
\hline N1 & $34(32)$ \\
\hline $\mathrm{N} 2$ & $6(6)$ \\
\hline \multicolumn{2}{|l|}{ Tumor type, n (\%) } \\
\hline Ductal & $59(56)$ \\
\hline Lobular & $25(24)$ \\
\hline Mixed & $18(17)$ \\
\hline Other $^{t}$ & $4(4)$ \\
\hline \multicolumn{2}{|l|}{ Tumor grade, n (\%) } \\
\hline 1 & $21(20)$ \\
\hline 2 & $58(55)$ \\
\hline 3 & $27(25)$ \\
\hline \multicolumn{2}{|l|}{ Biomarker status, n (\%) } \\
\hline $\mathrm{ER}^{+} \mathrm{PgR}^{+}$ & $75(71)$ \\
\hline $\mathrm{ER}^{+} \mathrm{PgR}^{-}$ & $30(28)$ \\
\hline $\mathrm{ER}^{-} \mathrm{PgR}^{+}$ & $1(1)$ \\
\hline HER2-negative & $97(91.5)$ \\
\hline HER2-positive & $8(7.6)$ \\
\hline HER2 unknown & $1(0.9)$ \\
\hline
\end{tabular}

Nine patients excluded from analysis because of withdrawal of consent $(n=7)$, ineligible $(n=1)$, and death unrelated to disease $(n=1)$. 
Mucinous, 2 (2\%); tubular, $1(1 \%)$; papillary, $1(1 \%)$.

ECOG, Eastern Cooperative Oncology Group performance status; ER, estrogen receptor; PgR, progesterone receptor. 
Table 2

Summary of Adverse Events that Occured During Preoperative Letrozole Treatment $(n=106)$

\begin{tabular}{lccccc}
\hline & \multicolumn{2}{c}{ Total } & & \multicolumn{2}{c}{ Grade $\mathbf{3}$} \\
\cline { 2 - 3 } \cline { 5 - 6 } Adverse event & $\mathbf{n}$ & $\%$ & & $\mathbf{n}$ & $\%$ \\
\hline Hot flashes & 68 & 64 & 0 & 0 \\
\hline Arthralgia & 48 & 45 & 3 & 3 \\
\hline Fatigue & 45 & 42 & 0 & 0 \\
\hline Night sweats & 13 & 12 & 0 & 0 \\
\hline Nausea and vomiting & 13 & 12 & 0 & 0 \\
\hline Gastrointestinal (all) & 53 & 50 & 0 & 0 \\
\hline Neurologic (all) & 46 & 57 & 0 & 0 \\
\hline Dermatologic (all) & 28 & 26 & 0 & 0 \\
\hline Infection (all) & 26 & 25 & 5 & 5 \\
\hline Metabolic (all) & 14 & 13 & 4 & 4 \\
\hline Cardiovascular (all) & 5 & 5 & 3 & 3 \\
\hline Hematologic (all) & 7 & 7 & 0 & 0 \\
\hline
\end{tabular}




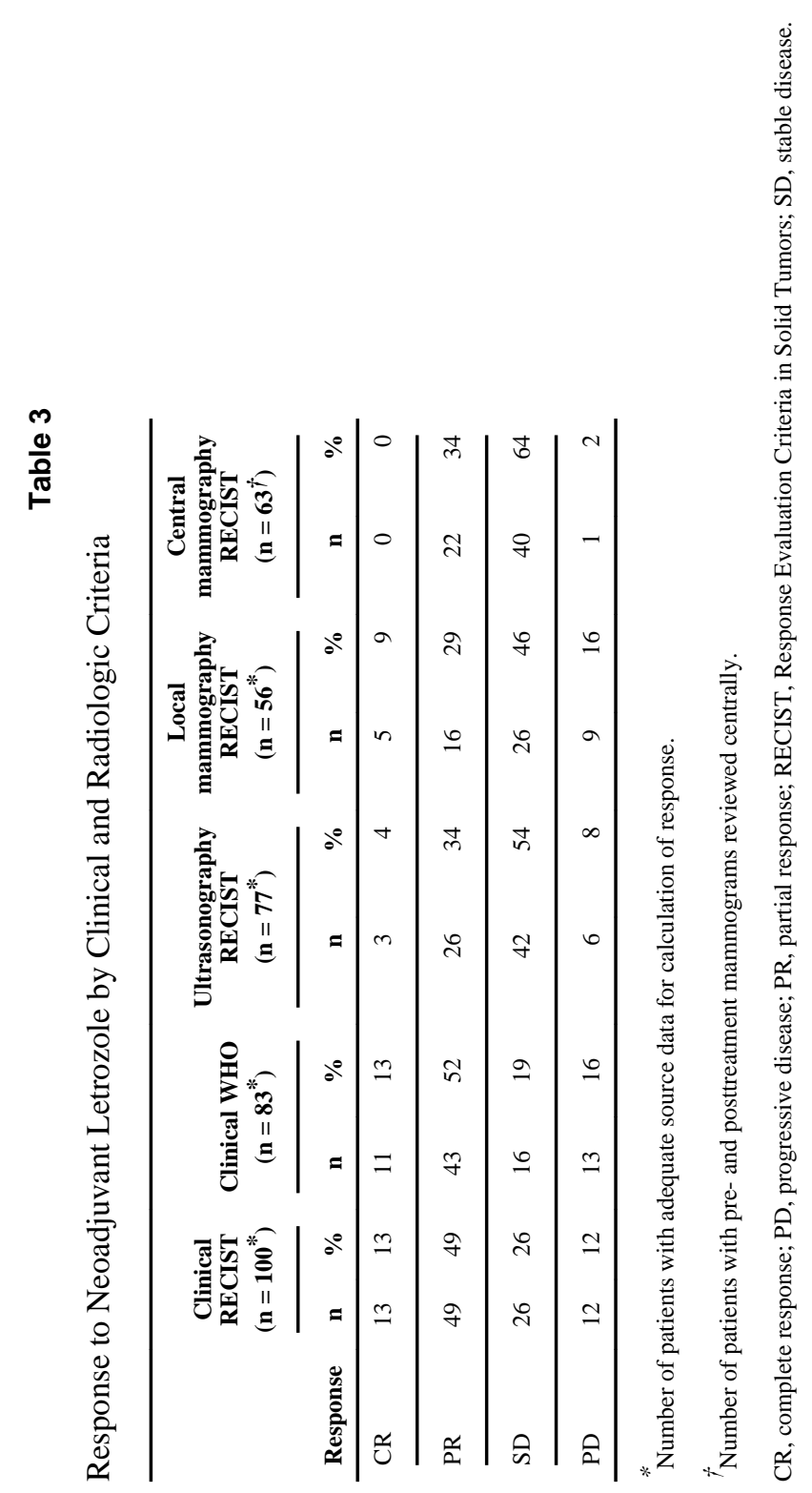

J Am Coll Surg. Author manuscript; available in PMC 2013 June 17. 
Table 4

Final Surgical Outcomes According to Baseline Surgical Category

\begin{tabular}{lcrrr}
\hline $\begin{array}{l}\text { Preoperative } \\
\text { assessment }\end{array}$ & $\mathbf{n}^{*}$ & BCS & Mastectomy & Conversion (\%) \\
\hline Marginal for BCS & 46 & 30 & 16 & $30 / 46(65)$ \\
\hline Ineligible for BCS & 39 & 15 & 24 & $15 / 39(38)$ \\
\hline Inoperable & 11 & 3 & 8 & $3 / 11(27)$ \\
\hline Total & 96 & 48 & 48 & $48 / 96(50)$ \\
\hline
\end{tabular}

One patient refused operation; two patients were not operable because of unassociated illness and comorbidities and seven patients with Response Evaluation Criteria in Solid Tumors progressive disease received neoadjuvant chemotherapy followed by breast-conserving surgery $(n=3)$ or mastectomy $(\mathrm{n}=4)$.

BCS, breast-conserving surgery. 
Table 5

Pathological Stage after Completion of Neoadjuvant Letrozole Therapy

\begin{tabular}{|c|c|}
\hline Characteristic & Value \\
\hline Pathologic tumor size $(\mathrm{cm})$, median (range) & $2.1(0.0-12.2)$ \\
\hline \multicolumn{2}{|l|}{ T stage, $\mathrm{n}(\%)$} \\
\hline T0 & $1(1)$ \\
\hline $\mathrm{T} 1$ & $39(41)$ \\
\hline $\mathrm{T} 2$ & $39(41)$ \\
\hline $\mathrm{T} 3$ & $13(14)$ \\
\hline $\mathrm{T} 4$ & $4(4)$ \\
\hline \multicolumn{2}{|l|}{$\mathrm{N}$ stage, $\mathrm{n}(\%)$} \\
\hline No & $39(41)$ \\
\hline N1 & $34(35)$ \\
\hline $\mathrm{N} 2$ & $13(14)$ \\
\hline N3 & $8(8)$ \\
\hline $\mathrm{Nx}$ & $2(2)$ \\
\hline \multicolumn{2}{|l|}{ Pathologic stage, n (\%) } \\
\hline 0 & $1(1)$ \\
\hline 1 & $21(22)$ \\
\hline $2 \mathrm{~A}$ & $22(23)$ \\
\hline $2 \mathrm{~B}$ & $20(21)$ \\
\hline $3 \mathrm{~A}$ & $18(19.0)$ \\
\hline 3B & $4(4)$ \\
\hline $3 \mathrm{C}$ & $8(8)$ \\
\hline Stage incomplete ${ }^{*}$ & $2(2)$ \\
\hline \multicolumn{2}{|l|}{ Tumor type, n (\%) } \\
\hline Ductal & $51(53)$ \\
\hline Lobular & $30(31)$ \\
\hline Mixed & $11(12)$ \\
\hline Other $^{t}$ & $3(3)$ \\
\hline No residual tumor & $1(1)$ \\
\hline \multicolumn{2}{|l|}{ Tumor grade, n (\%) } \\
\hline 1 & $30(32)$ \\
\hline 2 & $57(59)$ \\
\hline 3 & $8(8)$ \\
\hline No residual tumor & $1(1)$ \\
\hline
\end{tabular}


Table 6

Univariate Analysis of Factors that Influenced the Use of Breast Conservation Surgery vs Mastectomy

\begin{tabular}{|c|c|c|c|c|}
\hline Feature & BCS & Mastectomy & p Value & Relative risk ${ }^{*}(95 \%$ CI $)$ \\
\hline Age (y), mean (SD) & $66.7(10.05)$ & $66.6(10.1)$ & 0.97 & \\
\hline \multicolumn{5}{|l|}{ Biomarker } \\
\hline $\mathrm{ER}^{+} / \mathrm{PR}^{+}$ & 34 & 35 & 0.82 & \\
\hline $\mathrm{ER}^{+} / \mathrm{PR}^{-}$ & 14 & 13 & & \\
\hline \multicolumn{5}{|l|}{ Pre-AI cT stage } \\
\hline cT2 & 36 & 16 & & \\
\hline сT3/4 & 12 & 32 & $<0.0001$ & $2.4(1.5-3.7)$ \\
\hline \multicolumn{5}{|l|}{ Clinical N stage } \\
\hline $\mathrm{cNO}$ & 31 & 29 & 0.67 & \\
\hline $\mathrm{cN} 1 / 2$ & 17 & 19 & & \\
\hline \multicolumn{5}{|l|}{ Histologic subtype } \\
\hline Ductal & 39 & 33 & & \\
\hline Lobular $^{\dagger}$ & 9 & 15 & 0.16 & \\
\hline \multicolumn{5}{|l|}{ Pre-AI surgical status } \\
\hline Marginal BCS & 30 & 16 & & \\
\hline Ineligible $\mathrm{BCS}{ }^{t}$ & 15 & 24 & 0.0042 & $1.84(1.18-2.88)$ \\
\hline Inoperable & 3 & 8 & & \\
\hline \multicolumn{5}{|l|}{ Clinical response } \\
\hline $\mathrm{PR}+\mathrm{CR}$ & 35 & 26 & & \\
\hline $\mathrm{SD}+\mathrm{PD}$ & 11 & 19 & 0.0632 & $1.49(1.0-2.2)$ \\
\hline \multicolumn{5}{|l|}{ Post-AI cT stage } \\
\hline cT0/1 & 24 & 9 & & \\
\hline $\mathrm{cT} 2 / 3 / 4$ & 24 & 39 & 0.0013 & $2.27(1.26-4.1)$ \\
\hline \multicolumn{5}{|l|}{ Pathologic T stage } \\
\hline $\mathrm{p}<\mathrm{T} 2$ & 47 & 32 & & \\
\hline pT3,4 & 1 & 16 & $<0.0001$ & $2.32(1.73-3.11)$ \\
\hline \multicolumn{5}{|l|}{ Pathologic N stage } \\
\hline pNO & 28 & 11 & & \\
\hline $\mathrm{pN} 1-3$ & 20 & 37 & 0.0004 & $2.3(1.35-3.9)$ \\
\hline \multicolumn{5}{|c|}{$\begin{array}{l}\text { Relative risks and } 95 \% \mathrm{CI} \text { of having a mastectomy were calculated with a feature's lower level } \\
\text { Includes ductal with lobular features. }\end{array}$} \\
\hline $\mathrm{cT}$ and $\mathrm{cN}$ indicates $\mathrm{cl}$ & $\mathrm{al} \mathrm{T}$ and $\mathrm{N}$ sta & ge. & & \\
\hline
\end{tabular}

AI, aromatase inhibitor; BCS, breast-conserving surgery; CR, complete response; ER, estrogen receptor; PD, progressive disease; PR, progesterone receptor; $\mathrm{SD}$, stable disease. 
Table 7

Multivariable Analysis to Identify Independent Factors that Influenced the Use of Breast Conservation Surgery versus Mastectomy

\begin{tabular}{lcc}
\hline Factor & p Value & $\begin{array}{c}\text { Odds ratio } \\
\text { (95\% Wald CI) }\end{array}$ \\
\hline Pre-AI clinical T stage (3/4 versus 2) & 0.012 & $3.67(1.3-10.1)$ \\
\hline Pre-AI surgical status (others versus marginal) & 0.019 & $3.21(1.2-8.5)$ \\
\hline Post-AI cT stage (2/3/4 versus 1/0) & 0.029 & $3.3(1.1-9.4)$ \\
\hline
\end{tabular}

AI, aromatase inhibitor. 